\title{
HYPERSURFACES WITH TWO PRINCIPAL CURVATURES IN $\mathbb{S}^{n+1}$.
}

\author{
Sebastião C. de Almeida Aldir Brasil Jr.
}

\begin{abstract}
In this paper we consider compact oriented hypersurfaces with constant mean curvature immersed in the Euclidean sphere $\mathbb{S}^{n+1}$. Recently, restricting to hypersurfaces $M$ with two distinct principal curvatures with multiplicities 1 and $n-1$ respectively, the authors jointly with L.J.Alias $[\mathrm{AAB}]$, obtained an integral inequality involving the square of the norm of the second fundamental form of $M$. The equality holds only if $M$ is the $H(r)$-torus. In the present paper we show that under the same conditions, $M$ is the locus of a codimension-one submanifold moving along one of the lines of principal curvatures of $M$. In the process we give a sketch of the integral inequality in $[\mathrm{AAB}]$ and establish a certain ordinary differential equation involving the invariants of $M$.
\end{abstract}

\section{Introduction.}

Let $M$ be a closed hypersurface of the $(n+1)$-dimensional unit Euclidean sphere $\mathbb{S}^{n+1}$. As usual, let $S$ be the square of the length of the second fundamental form $A$ of $M$. In [HV], Hasanis and Vlachos proved that if $M$ is a compact minimal hypersurface with two principal curvatures, one of them with multiplicity $k=1$ and $S \geq n$ then $S=n$ and $M$ is a Clifford torus. In [BBCL], the second author jointly with Barbosa, Costa and Lazaro obtained a generalization of the above result with no hypothesis on the constancy of the mean curvature. They obtained a characterization for the $H(r)$-tori $\mathbb{S}^{n-1}(r) \times \mathbb{S}^{1}\left(\sqrt{1-r^{2}}\right)$ with $r^{2} \geq(n-1) / n$. More precisely:

Theorem 1.1. [BBCL] Let $M$ be a compact oriented hypersurface immersed in the sphere $\mathbb{S}^{n+1}$, with two distinct principal curvatures $\lambda$ and $\mu$ with multiplicities 
1 and $n-1$, respectively. Let $\Phi=A-H I$ be the traceless second fundamental form of $M$. Suppose in addition that $n \geq 3$ and $|\Phi|^{2} \geq C_{H}$, where

$$
C_{H}=n+\frac{n\left(n^{2}-2 n+2\right) H^{2}}{2(n-1)}+\frac{n(n-2)|H|}{2(n-1)} \sqrt{n^{2} H^{2}+4(n-1)} .
$$

Then $H$ is constant, $|\Phi|^{2}=C_{H}$ and $M$ is isometric to an $H(r)$-torus $\mathbb{S}^{n-1}(r) \times$ $\mathbb{S}^{1}\left(\sqrt{1-r^{2}}\right)$ with $r^{2} \geq(n-1) / n$.

Recently, Perdomo $[\mathrm{P}]$ and Wang [W], simultaneous and independently, proved that if $M \subset \mathbb{S}^{n+1}$ is a compact minimal hypersurface with two principal curvatures, one of them with multiplicity $k=1$ then

$$
\int_{M}\left(|A|^{2}-n\right) \leq 0
$$

with equality if and only if $M$ is a Clifford hypersurface. Recently, the authors jointly with L.J. Alias [AAB] extended the result of Perdomo and Wang for compact hypersurfaces with constant mean curvature and obtained a new characterization of the $H(r)$-torus $\mathbb{S}^{n-1}(r) \times \mathbb{S}^{1}\left(\sqrt{1-r^{2}}\right)$. Explicitly, we have the following result (Theorem 3 and Corollary 4 in [AAB]).

Theorem 1.2. Let $M$ be a compact oriented hypersurface immersed in the sphere $\mathbb{S}^{n+1}$ with constant mean curvature $H$. Suppose in addition that $M$ has two distinct principal curvatures $\lambda$ and $\mu$ with multiplicities $(n-1)$ and 1 , respectively and let $c= \pm 1$ be the sign of the difference $\lambda-\mu$. If $\Phi=A-H I$ is the traceless second fundamental form tensor of $M$ and $P_{H}$ is the Alencar-Do Carmo polynomial

$$
P_{H}(x)=x^{2}+\frac{n(n-2)}{\sqrt{n(n-1)}} H x-n\left(1+H^{2}\right)
$$

then

$$
\int_{M} P_{H}(c|\Phi|)=-\left.\frac{(n-2)}{n} \int_{M}|\nabla \ln | \Phi\right|^{2} \leq 0,
$$

with equality only if $M$ is an $H(r)$-torus $\mathbb{S}^{n-1}(r) \times \mathbb{S}^{1}\left(\sqrt{1-r^{2}}\right)$. 
In [O], Otsuki gave necessary conditions for a minimal hypersurface of the sphere to be a product of spheres. He showed that if a compact minimal hypersurface has two principal curvatures with multiplicities greater than one, then the hypersurface must be a Clifford hypersurface. On the other hand, by studying a certain ordinary differential equation he was led to the construction of infinitely many non standard minimal hypersurfaces. Those hypersurfaces have only two principal curvatures with one of them of constant multiplicity $k=1$. By making use of a technique similar to the method of Otsuki, we prove the following result.

Theorem 1.3. Let $M$ be an $n$-dimensional hypersurface of $\mathbb{S}^{n+1}(1)$ with constant mean curvature $H$ and two distinct principal curvatures $\mu$ and $\lambda$. Suppose in addition that $n \geq 3$ and the principal vectors corresponding to $\mu$ is a 1 dimensional space. Then $M$ is the locus of a codimension-one submanifold $X^{n-1}(s)$ moving along the line of curvature determined by $\mu$. Locally $X^{n-1}(s)$ is isometric to an $(n-1)$-dimensional sphere $\mathbb{S}^{n-1}\left(c_{s}\right)$ of constant curvature

$$
c_{s}=\left\{\frac{d\left(\ln |H-\lambda|^{\frac{1}{n}}\right)}{d s}\right\}^{2}+\lambda^{2}+1 .
$$

The function $w=1 /(\lambda-H)^{1 / n}$ satisfies the ordinary differential equation

$$
\frac{d^{2} w}{d s^{2}}-w\left(\frac{n-1}{w^{2 n}}+\frac{n-2}{w^{n}} H-H^{2}-1\right)=0,
$$

and $\mathbb{S}^{n-1}\left(c_{s}\right)$ is the intersection of the unit sphere $\mathbb{S}^{n+1}(1)$ with a $n$-dimensional linear subspace $E^{n}(s)$ of the Euclidean space $\mathbb{R}^{n+2}$ which are parallel to a fixed $E^{n}$.

In this note we give a sketch of the proof of Theorem 1.2 and present a complete proof of Theorem 1.3.

\section{Preliminaries}

Let $M$ be a compact hypersurface with constant mean curvature $H$ immersed in $\mathbb{S}^{n+1}$. As in $[\mathrm{AAB}]$, choose a local orthornormal frame field $E_{1}, \ldots, E_{n}$ in a 
neighborhood $U$ of $M$ and let $\omega_{1}, \ldots, \omega_{n}$ be its dual coframe. As is well known, there are smooth 1 -forms $\omega_{i j}$ on $U$ uniquely determined by the equations

$$
d \omega_{i}=-\sum_{j=1}^{n} \omega_{i j} \wedge \omega_{j}, \quad \omega_{i j}+\omega_{j i}=0 .
$$

The square of the length of the second fundamental form

$$
A=\sum_{i, j=1}^{n} h_{i j} \omega_{i} \otimes \omega_{j},
$$

is given by $S=\sum_{i, j} h_{i j}^{2}$. Note that $h_{i j}=h_{j i}$ and

$$
H=\frac{1}{n} \sum_{i=1}^{n} h_{i i} .
$$

The covariant derivative of $h_{i j}$ is given by

$$
\sum_{k} h_{i j k} \omega_{k}=d h_{i j}-\sum_{m} h_{i m} \omega_{m j}-\sum_{m} h_{m j} \omega_{m i}
$$

It is well known that $h_{i j k}$ is symmetric in all indices. From now we will assume that $M^{n}$ is a compact hypersurface with constant mean curvature having everywhere two distinct principal curvatures $\lambda$ and $\mu$ with multiplicities $n-k$ and $k$, respectively. We now recall a classical result of Otsuki [O].

Proposition 2.1. Let $M$ be a hypersurface in $\mathbb{S}^{n+1}$ such that the multiplicities of its principal curvatures are constant. Then the distribution $D_{\lambda}$ of the space of principal vectors corresponding to each principal curvature $\lambda$ is completely integrable. In particular, if the multiplicity of a principal curvature is greater than 1, then this principal curvature is constant on each of the integral leaves of the corresponding distribution .

A consequence of this result is the following lemma:

Lemma 2.2. Let $M^{n}$ be a compact oriented hypersurface in $\mathbb{S}^{n+1}$ with constant mean curvature and two principal curvatures $\lambda$ and $\mu$, with multiplicities $n-$ $k$ and $k$ respectively. If $1<k<n-1$, then $M$ is isometric to $\mathbb{S}^{n-k}(r) \times$ $\mathbb{S}^{k}\left(\sqrt{1-r^{2}}\right)$. 
Proof. We may choose a local orthonormal basis $E_{1}, \ldots, E_{k}, E_{k+1}, \ldots, E_{n}$ such that for $1 \leq i \leq k$ and $k+1 \leq j \leq n$,

$$
\begin{aligned}
& E_{i} \in D_{\lambda}=\left\{v \in T_{p} M: p \in M, A v=\lambda v\right\} \\
& E_{j} \in D_{\mu}=\left\{v \in T_{p} M: p \in M A v=\mu v\right\} .
\end{aligned}
$$

By Proposition 2.1 above, we have $E_{i}(\lambda)=E_{j}(\mu)=0$ for $i \leq k$ and $k+1 \leq j$. As $n H=k \lambda+(n-k) \mu$ is a constant function, it follows that

$$
k E_{j}(\lambda)=(k-n) E_{j}(\mu)=0
$$

Therefore $\mu$ and $\lambda$ are constant and $M$ is an isoparametric hypersurface. Note that since $M$ is compact, then $M$ is isometric to $\mathbb{S}^{n-k}(r) \times \mathbb{S}^{k}\left(\sqrt{1-r^{2}}\right)$.

\section{Sketch of the proof of Theorem 1.2}

As in $[\mathrm{AAB}]$ we introduce the traceless second fundamental form $\Phi=A-H I$. Taking into account the different choice of sign in the definition of $\Phi$ in Alencar and do Carmo $[\mathrm{AC}]$ we have the Simons formula:

$$
\frac{1}{2} \Delta|\Phi|^{2}=|\nabla \Phi|^{2}+|\Phi|^{2}\left(n\left(1+H^{2}\right)-|\Phi|^{2}\right)+n H \operatorname{tr}\left(\Phi^{3}\right) .
$$

In the following lemma we are going to evaluate the Laplacian of $\ln |\Phi|$. It turns out that $\Delta \ln |\Phi|$ depends on the polynomial

$$
P_{H}(x)=x^{2}+\frac{n(n-2) H}{\sqrt{n(n-1)}} x-n\left(1+H^{2}\right) .
$$

Lemma 3.1. Let $M$ be a compact oriented hypersurface with constant mean curvature $H$ immersed in $\mathbb{S}^{n+1}$ having two principal curvatures $\lambda$ and $\mu$, with multiplicities $n-1$ and 1 respectively. Then

$$
\Delta \ln |\Phi|=\frac{1}{|\Phi|^{2}}\left(|\nabla \Phi|^{2}-\left.2|\nabla| \Phi\right|^{2}\right)-P_{H}(c|\Phi|),
$$

where $c= \pm 1$ is the sign of the difference $\lambda-\mu$. 
Proof. Note that

$$
\begin{aligned}
|\Phi|^{2} \mid \Delta(\ln |\Phi|) & =|\Phi|^{2}\left(\frac{1}{|\Phi|} \Delta|\Phi|-\frac{1}{|\Phi|^{2}}|\nabla| \Phi||^{2}\right) \\
& =|\Phi| \Delta|\Phi|-\left.|\nabla| \Phi\right|^{2} \\
& =\frac{1}{2} \Delta|\Phi|^{2}-\left.2|\nabla| \Phi\right|^{2} .
\end{aligned}
$$

A straightforward computation gives,

$$
\operatorname{tr}\left(\Phi^{3}\right)=-\frac{(n-2)}{\sqrt{n(n-1)}} c|\Phi|^{3} .
$$

Using Simons formula, one gets

$$
\Delta \ln |\Phi|=\frac{1}{|\Phi|^{2}}\left(|\nabla \Phi|^{2}-\left.2|\nabla| \Phi\right|^{2}\right)-P_{H}(c|\Phi|) .
$$

Lemma 3.2. Let $M^{n}, n \geq 3$, be a compact oriented hypersurface with constant mean curvature $H$ immersed in $\mathbb{S}^{n+1}$ having two principal curvatures $\lambda$ and $\mu$ with multiplicities $n-1$ and 1 respectively, then

$$
|\nabla \Phi|^{2}=\left.\frac{n+2}{n}|\nabla| \Phi\right|^{2}
$$

Proof. Let $E_{1}, \ldots, E_{n}$ be a local orthonormal frame field so that $\Phi\left(E_{n}\right)=\tilde{\mu} E_{n}$, and

$$
\Phi\left(E_{i}\right)=\tilde{\lambda} E_{i}
$$

for all $1 \leq i \leq n-1$. Note that

$$
|\nabla \Phi|^{2}=|\nabla A|^{2}=\sum_{i, j, k} h_{i j k}^{2}
$$

with

$$
\sum_{k} h_{i j k} \omega_{k}=d h_{i j}-\sum_{m} h_{i m} \omega_{m j}-\sum_{m} h_{m j} \omega_{m i}
$$


From this and a straightforward computations gives

$$
|\nabla \Phi|^{2}=(n-1)(n+2) \frac{\left.|\nabla| \Phi\right|^{2}}{n(n-1)}=\frac{(n+2)}{n}|\nabla| \Phi \|^{2} .
$$

Theorem 1.2 follows from Lemma 3.1 and Lemma 3.2. In fact,

$$
\begin{aligned}
\Delta \ln |\Phi| & =\frac{1}{|\Phi|^{2}}\left(|\nabla \Phi|^{2}-2|\nabla| \Phi||^{2}\right)-P_{H}(c|\Phi|) \\
& =\left.\frac{2-n}{n|\Phi|^{2}}|\nabla| \Phi\right|^{2}-P_{H}(c|\Phi|) \\
& =\left.\frac{2-n}{n}|\nabla \ln | \Phi\right|^{2}-P_{H}(c|\Phi|) .
\end{aligned}
$$

Integrating now over $M$ we conclude that

$$
\int_{M} P_{H}(c|\Phi|)=-\frac{(n-2)}{n} \int_{M}|\nabla \ln | \Phi \|^{2} .
$$

This finishes the proof of Theorem 1.2.

As a new application, in the minimal case we may use the same technique to retrieve Hasanis-Vlachos result:

Theorem 3.3. Let $M$ be a compact minimal hypersurface of $\mathbb{S}^{n+1}$ with two distinct principal curvatures with multiplicities 1 and $n-1$ respectively. If $S \geq n$ is the square of the length of the second fundamental form, then $S=n$ and $M$ is a Clifford torus.

In fact, by making $H=0$ in Lemma 3.1, we note that

$$
2 n \Delta \ln S=4 n(n-S)+(2-n)|\nabla \ln S|^{2} .
$$

Hasanis-Vlachos theorem will follow as a consequence. 


\section{Proof Theorem 1.3}

We will assume that the principal curvatures of $M$ are given by $\lambda_{1}=\cdots=$ $\lambda_{n-1}=\lambda$ and $\lambda_{n}=\mu$ and choose a local orthonormal frame $E_{1}, \ldots, E_{n-1}, E_{n}$ such that for $1 \leq i \leq n-1$

$$
\begin{gathered}
E_{i} \in D_{\lambda}=\left\{v \in T_{p} M: p \in M, A v=\lambda v\right\} \\
E_{n} \in D_{\mu}=\left\{v \in T_{p} M: p \in M, A v=\mu v\right\} .
\end{gathered}
$$

Recall that, for all $i, j, k \in I=\{1,2, \ldots, n\}$,

$$
\sum_{k} h_{i j k} \omega_{k}=d h_{i j}-\sum_{m} h_{i m} \omega_{m j}-\sum_{m} h_{m j} \omega_{m i}
$$

From this it follows that

$$
d \mu\left(E_{k}\right)=(n-1) d \lambda\left(E_{k}\right)=0
$$

for all $k \in I-\{n\}$. To see this, just choose $m \in I-\{k, n\}$ and note that

$$
d \lambda\left(E_{k}\right)=h_{m m k}=h_{m k m}=\left(h_{k k}-h_{m m}\right) w_{m k}\left(E_{m}\right)=0 .
$$

We also note that for $i \neq n$,

$$
\begin{aligned}
\omega_{n i} & =\sum_{k=1}^{n} \omega_{n i}\left(E_{k}\right) \omega_{k}=\sum_{k=1}^{n} \frac{h_{n i k}}{h_{i i}-h_{n n}} \omega_{k} \\
& =\frac{h_{n i n}}{h_{i i}-h_{n n}} \omega_{n}+\frac{h_{n i i}}{h_{i i}-h_{n n}} \omega_{i}+\sum_{i \neq k=1}^{n-1} \frac{h_{n i k}}{h_{i i}-h_{n n}} \omega_{k} \\
& =\frac{h_{n n i}}{\lambda-\mu} \omega_{n}+\frac{h_{i i n}}{\lambda-\mu} \omega_{i}+\sum_{i \neq k=1}^{n-1} \frac{h_{i k n}}{\lambda-\mu} \omega_{k} \\
& =\frac{\left\langle\nabla \mu, E_{i}\right\rangle}{\lambda-\mu} \omega_{n}+\frac{\left\langle\nabla \lambda, E_{n}\right\rangle}{\lambda-\mu} \omega_{i}+\sum_{i \neq k=1}^{n-1} \frac{\left(h_{k k}-h_{i i}\right) \omega_{i k}\left(E_{n}\right)}{\lambda-\mu} \omega_{k} \\
& =\frac{\left\langle\nabla \lambda, E_{n}\right\rangle}{\lambda-\mu} \omega_{i} .
\end{aligned}
$$

Now we set $w=|\lambda-H|^{-1 / n}$. By changing the sign of $H$ we may assume that $\lambda>0$. Note that $\lambda-\mu=n(\lambda-H) \neq 0$. If $\lambda-H<0$, then $0<\lambda<\mu$. Since 
the sectional curvature of $M$ is greater than or equal to zero then by Hartman theorem $M$ would be a totally umbilical hypersurface, which is impossible. From now on we assume that $\lambda-H>0$. With this notation, we have

$$
\omega_{i n}=-\frac{d}{d s}\left(\ln |\lambda-H|^{\frac{1}{n}}\right) \omega_{i}=\frac{d(\ln w)}{d s} \omega_{i},
$$

where $\frac{\partial}{\partial s}=\alpha^{\prime}$ and $\alpha(s)$ is the line of curvature corresponding to the principal curvature $\mu$ with $\alpha^{\prime}=E_{n}$. Using the fact $d \lambda\left(E_{k}\right)=0$, for all $k \in I-\{n\}$ and a straightforward computation we get

$$
\begin{aligned}
d \omega_{i n} & =(\ln w)^{\prime \prime} d s \wedge \omega_{i}-(\ln w)^{\prime} \sum_{k=1}^{n-1} \omega_{i k} \wedge \omega_{k}-(\ln w)^{\prime} \omega_{i n} \wedge \omega_{n} \\
& =-(\ln w)^{\prime \prime} \omega_{i} \wedge \omega_{n}-(\ln w)^{\prime} \sum_{k=1}^{n-1} \omega_{i k} \wedge \omega_{k}-\left[(\ln w)^{\prime}\right]^{2} \omega_{i} \wedge \omega_{n}
\end{aligned}
$$

On the other hand, from the structure equations of $\mathbb{S}^{n+1}$, we also have

$$
\begin{aligned}
d \omega_{i n} & =-\sum_{k=1}^{n-1} \omega_{i k} \wedge \omega_{k n}-\omega_{i n+1} \wedge \omega_{n+1 n}+\omega_{i} \wedge \omega_{n} \\
& =-(\ln w)^{\prime} \sum_{k=1}^{n-1} \omega_{i k} \wedge \omega_{k}+(1+\lambda \mu) \omega_{i} \wedge \omega_{n} .
\end{aligned}
$$

In the last equality we use the fact that $\omega_{n+1 n}=\mu \omega_{n}$ and $\omega_{i n+1}=-\lambda \omega_{i}$, for $1 \leq i \leq n-1$. Comparing the equations we have

$$
\left.(\ln w)^{\prime \prime}+\left[(\ln w)^{\prime}\right]^{2}\right]+\lambda \mu+1=0 .
$$

Since $\lambda \mu=n H \lambda-(n-1) \lambda^{2}$, we have

$$
\left.(\ln w)^{\prime \prime}+\left[(\ln w)^{\prime}\right]^{2}\right]-(n-1) \lambda^{2}+n H \lambda+1=0
$$

or equivalently

$$
\frac{d^{2} w}{d s^{2}}-\frac{n-1}{w^{2 n-1}}-\frac{n-2}{w^{n-1}} H+\left(H^{2}+1\right) w=0 .
$$

Integrating the equation above, we have

$$
\left(\frac{d w}{d s}\right)^{2}+\frac{1}{w^{2 n-2}}+2 H \frac{1}{w^{n-2}}+\left(H^{2}+1\right) w^{2}=C,
$$


where $\mathrm{C}$ is the constant of integration. Note that

$$
d E_{i}=-\sum_{j=1}^{n-1} \omega_{i j} E_{j}-\omega_{i n} E_{n}-\omega_{i n+1} E_{n+1}-\omega_{i} E_{n+2}=-\sum_{j=1}^{n-1} \omega_{i j} E_{j}+Z \omega_{i}
$$

where $Z$ is the vector field given by

$$
Z=(-\ln w)^{\prime} E_{n}+\lambda E_{n+1}-E_{n+2}
$$

Now we consider the frame field $b=\left(x, E_{1}, \ldots, E_{n+1}\right)$ in $\mathbb{R}^{n+2}$ where $x=$ $E_{n+2}$. Note that $\bmod \left(E_{1}, \ldots, E_{n-1}\right)$,

$$
\begin{aligned}
d Z & =-(\ln w)^{\prime \prime} w_{n} E_{n}-(\ln w)^{\prime}\left(\mu \omega_{n} E_{n+1}-\omega_{n} E_{n+2}\right)+\lambda^{\prime} \omega_{n} E_{n+1}-\lambda \mu \omega_{n} E_{n}-\omega_{n} E_{n} \\
& =-\left[(\ln w)^{\prime \prime}+\lambda \mu+1\right] \omega_{n} E_{n}-\left[\mu(\ln w)^{\prime}-\lambda^{\prime}\right] \omega_{n} E_{n+1}+(\ln w)^{\prime} \omega_{n} E_{n+2} \\
& =\left[(\ln w)^{\prime}\right]^{2} \omega_{n} E_{n}-\lambda(\ln w)^{\prime} \omega_{n} E_{n+1}+(\ln w)^{\prime} \omega_{n} E_{n+2} \\
& =-(\ln w)^{\prime} \omega_{n} Z .
\end{aligned}
$$

Therefore

$$
Z(s)=\left[\frac{H-\lambda(s)}{H-\lambda\left(s_{0}\right)}\right]^{1 / n} Z\left(s_{0}\right)
$$

for some fixed value $s_{0}$ of the parameter $s$. From equation (4.2) it follows that

$$
d\left(E_{1} \wedge \cdots \wedge E_{n-1} \wedge Z\right)=-(\ln w)^{\prime} E_{1} \wedge \cdots \wedge E_{n-1} \wedge Z d s
$$

Then the $n$-vector field $W=E_{1} \wedge \cdots \wedge E_{n-1} \wedge Z$ depends only on the parameter $s$ and it is given by

$$
W(s)=\left[\frac{H-\lambda(s)}{H-\lambda\left(s_{0}\right)}\right]^{1 / n} W\left(s_{0}\right) .
$$

We know that the integral leaves of the $C^{\infty}$ - distribution

$$
D_{\lambda}=\left\{v \in T_{p} M: p \in M, A v=\lambda v\right\}
$$


is a codimension-one submanifold $X^{n-1}(s)$ moving along the line of curvature corresponding to the principal curvature $\mu$. On the other hand, equation (4.4) shows that the $n$-vector $W$ is constant along $X^{n-1}(s)$. As a consequence $X^{n-1}(s)$ is contained in the $n$-dimensional linear space $E^{n}(s)$ determined by $W(s)$. Note that

$$
d \omega_{i j}+\sum_{k=1}^{n-1} \omega_{i k} \wedge \omega_{k j}=-\omega_{i n} \wedge \omega_{n j}-\omega_{i n+1} \wedge \omega_{n+1, j}+\omega_{i} \wedge \omega_{j}=c \omega_{i} \wedge \omega_{j} .
$$

where $c$ is equal to a constant

$$
c_{s}=|Z|^{2}=\left[(\ln w)^{\prime}\right]^{2}+\lambda^{2}+1,
$$

on each leaf $X^{n-1}(s)$ of the distribution. It follows that locally $X^{n-1}(s)$ is isometric to an $(n-1)$-dimensional sphere $\mathbb{S}^{n-1}\left(c_{s}\right)$ of constant curvature $c_{s}$. The function $w=1 /(\lambda-H)^{1 / n}$ satisfies the ordinary differential equation

$$
\frac{d^{2} w}{d s^{2}}-w\left(\frac{n-1}{w^{2 n}}+\frac{n-2}{w^{n}} H-H^{2}-1\right)=0,
$$

and $\mathbb{S}^{n-1}\left(c_{s}\right)$ is the intersection of the unit sphere $\mathbb{S}^{n+1}(1)$ with a $n$-dimensional linear subspace $E^{n}(s)$ of the Euclidean space $\mathbb{R}^{n+2}$ which are parallel to the fixed $n$-dimensional linear subspace $E^{n}\left(s_{0}\right)$ in $\mathbb{R}^{n+2}$.

Acknowledgments. The authors would like to thank the Professor Luis J. Alias for suggestions and hospitality at Universidad de Murcia. The second author was partially supported by CAPES BEX 032402/7.

\section{References}

[AAB] Alías, L., Almeida, S. C., Brasil, A.C, Hypersurfaces with constant mean curvature and two principal curvatures in $\mathbb{S}^{n+1}$, Anais da Academia Brasileira de Cincias, 76, (2004), 489-497.

[AC] Alencar, H., do Carmo, M., Hypersurfaces with constant mean curvature in Spheres, Proc. of the AMS, 120 (4), (1981), 1223-1229. 
[BBCL] Barbosa, J.N., Brasil, A.C, Costa, E., Lazaro, I.C., Hypersurfaces of the Euclidean Sphere with nonnegative Ricci curvature, Arch. Math., 81, (2003), 335-341.

[CDK] Do Carmo, M., Chern, S., Kobayashi, S., Minimal submanifolds of the sphere with second fundamental form of constant lenght, Functional Analysis and related Fields, Springer Verlag, New York, (1970), 59-75.

[HV] Hasanis, T., Vlachos, T., A pinching theorem for minimal hypersurfaces in a sphere, Arch. Math., 75,(2000), 469-471.

[L] Lawson, H., Local rigidity theorems for minimal hypersurfaces, Ann. of Math., 89, (1969), 187-197.

[O] Otsuki, T., Minimal hypersurface in a Riemannian manifold of constant curvature, Amer. J. Math, 92, (1970), 145-173.

[P] Perdomo, O., Rigidity of minimal hypersurfaces of spheres with two principal curvatures, Arch. Math., 82 (2), (2003), 180-184.

[S] Simons, J., Minimal varieties in Riemannian manifolds, Ann. of Math., 88, (1968), 62-105.

[W] Wang, Q., Rigidity of Clifford minimal hypersurface, Monatschefte fur Mathematik, 140, (2), (2003), 163-167.

CAEN, Universidade Federal do Ceará

Av. da Universidade, 2700

$2^{o}$ andar - Benfica

60020-181, Fortaleza-CE, Brazil

Email: sebastc@caen.ufc.br
Departamento de Matemática, Univiversidade Federal de do Ceará Campus do Pici 60455-760, Fortaleza-CE, Brazil 